\title{
Washington photometry of five star clusters in the Large Magellanic Cloud ${ }^{\star}$
}

\author{
A. E. Piatti ${ }^{1}$, D. Geisler ${ }^{2}$, A. Sarajedini ${ }^{3}$, and C. Gallart ${ }^{4}$ \\ 1 Instituto de Astronomía y Física del Espacio, CC 67, Suc. 28, 1428, Ciudad de Buenos Aires, Argentina \\ e-mail: andres@iafe.uba.ar \\ 2 Grupo de Astronomía, Departamento de Astronomía, Universidad de Concepción, Casilla 160-C, Concepción, Chile \\ 3 Department of Astronomy, University of Florida, PO Box 112055, Gainesville, FL 32611, USA \\ 4 Instituto de Astrofísica de Canarias, Calle Vía Laćtea, 38200, La Laguna, Tenerife, Spain
}

Received 28 March 2009 / Accepted 12 May 2009

ABSTRACT

\begin{abstract}
Aims. We present CCD photometry in the Washington system $C$ and $T_{1}$ passbands down to $T_{1} \sim 22.5$ in the fields of NGC 1697 , SL 133, NGC 1997, SL 663, and OHSC 28, five mostly unstudied star clusters in the LMC.

Methods. Cluster radii were estimated from star counts in appropriate-sized boxes distributed throughout the entire observed fields. We perform a detailed analysis of the field star contamination and derive cluster colour-magnitude diagrams (CMDs). Based on the best fits of isochrones computed by the Padova group to the $\left(T_{1}, C-T_{1}\right)$ CMDs, the $\delta\left(T_{1}\right)$ index and the Standard Giant Branch procedure, we derive metallicities and ages for the five clusters. We combine our sample with clusters with ages and metallicities on a similar scale and examine relationships between position in the LMC, age and metallicity.

Results. With the exception of NGC 1697 (age $=0.7 \mathrm{Gyr},[\mathrm{Fe} / \mathrm{H}]=0.0 \mathrm{dex}$ ), the remaining four clusters are of intermediate-age (from 2.2 to $3.0 \mathrm{Gyr}$ ) and relatively metal-poor $([\mathrm{Fe} / \mathrm{H}]=-0.7 \mathrm{dex})$. The cluster and field age-metallicty realtions show evidence for a metallicity offset but do overlap, particularly on the upper envelope side of the cluster age-metallicity relation.

Conclusions. We confirm previous results that clusters younger than $\sim 1$ Gyr were formed during an outside-in process; this occurred after a burst of cluster formation that took place mainly in the outer disk and peaked at $\sim 2$ Gyr ago.
\end{abstract}

Key words. techniques: photometric - galaxies: individual: LMC - Magellanic Clouds - galaxies: star clusters stars: Hertzsprung-Russell (HR) and C-M diagrams

\section{Introduction}

The ages and abundances of Magellanic Cloud (MC) star clusters are prime indicators of their galaxy's chemical evolution and star formation history. In addition, these star clusters are important in a number of additional ways beyond the MCs themselves. Because of their richness and the fact that they inhabit a region of age-metallicity space not covered by the Milky Way clusters, they have become vital testbeds for theoretical models of stellar evolution at intermediate-age and moderately low metallicity (Ferraro et al. 1995; Whitelock et al. 2003; Gallart et al. 2003). However, the number of well studied clusters in the MCs is still a very small percentage of those that have been catalogued, and detailed investigations of even a handfull of clusters represents a significant improvement on our knowledge of the chemical enrichment history of these galaxies.

Bekki \& Chiba (2005) investigated the dynamical and chemical evolution of the Large Magellanic Cloud (LMC) from a theoretical standpoint as it has interacted with the Galaxy and the Small Magellanic Cloud (SMC). They suggest that tidal interactions have occured which have stimulated bursts of star and cluster formation at specific periods, namely during periods of closest approach. A second observational challenge has recently been proposed by Bekki (2008), who suggests that the

* Tables 2-6 are only available in electronic form at the CDS via anonymous ftp to cdsarc.u-strasbg.fr $(130.79 .128 .5)$ or via http://cdsweb.u-strasbg.fr/cgi-bin/qcat?J/A+A/501/585
MC system has a common halo produced either by a dynamical coupling that started $\sim 4$ Gyr ago or by a remnant of a small group of galaxies destroyed via tidal stripping by the Milky Way. However, note that accurate proper motions which are so crucial for accurately modeling the orbits of the SMC and LMC are still problematic (Kallivayalil et al. 2006).

In the observational arena, Grocholski et al. (2006) confirmed that LMC clusters lack the metallicity gradient typically seen in non-barred galaxies, although Carrera et al. (2006) showed that the mean $[\mathrm{Fe} / \mathrm{H}]$ of the LMC field decreases with distance from the bar. This is an important issue to explore further, since any interaction with the SMC and/or the Galaxy had to leave its imprint on the chemical history of the LMC. Grocholski et al. also found that clusters with $[\mathrm{Fe} / \mathrm{H}]>-1.0 \mathrm{dex}$ show a tight metallicity distribution $([\mathrm{Fe} / \mathrm{H}]=-0.48 \pm 0.09)$, with no tail towards solar metallicities, in contrast with previous work (see, for example, Olszewsky et al. 1991). Carrera et al. (2008) obtained Ca II triplet metallicities of red giant branch stars in four LMC fields at galactocentric distances from $\sim 3^{\circ}$ to $8^{\circ}$ and found that the metallicity distribution in each field showed a well defined peak, with a tail towards lower metallicities. The mean metallicities remain constant until $\sim 6^{\circ}([\mathrm{Fe} / \mathrm{H}] \approx$ -0.5 dex), while for the outermost field the metallicity is subtantially lower $([\mathrm{Fe} / \mathrm{H}] \approx-0.8 \mathrm{dex})$.

Recently, Gallart et al. (2008) have presented evidence that, while the oldest field star population is coeval throughout the LMC disk, the age of the youngest component of the dominant 
Table 1. Observation log of selected clusters.

\begin{tabular}{|c|c|c|c|c|c|c|c|c|c|}
\hline 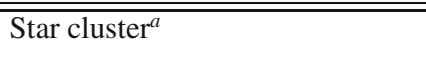 & $\begin{array}{c}\alpha_{2000} \\
(\mathrm{~h} \mathrm{~m} \mathrm{~s})\end{array}$ & $\begin{array}{l}\delta_{2000} \\
\left({ }^{\circ},{ }^{\prime \prime}\right)\end{array}$ & $\begin{array}{l}l \\
\left({ }^{\circ}\right)\end{array}$ & $\begin{array}{l}b \\
\left({ }^{\circ}\right)\end{array}$ & Date & Filter & $\begin{array}{c}\text { Exposure } \\
\text { (s) }\end{array}$ & Airmass & $\begin{array}{c}\text { Seeing } \\
\left({ }^{\prime \prime}\right)\end{array}$ \\
\hline \multirow[t]{2}{*}{ NGC 1697, SL 44, ESO 56-SC05 } & 44845 & -683845 & 280.01 & -36.20 & 1999 Dec. 29 & $\bar{C}$ & 1800 & 1.36 & 1.4 \\
\hline & & & & & & $R$ & 600 & 1.39 & 1.4 \\
\hline \multirow[t]{2}{*}{ SL 133, LW 99, KMHK 337} & 45721 & -652035 & 275.79 & -36.27 & 1999 Dec. 29 & $C$ & 1800 & 1.38 & 1.3 \\
\hline & & & & & & $R$ & 600 & 1.44 & 1.3 \\
\hline \multirow[t]{2}{*}{ NGC 1997, SL 520, LW 226} & 53012 & -631422 & 272.66 & -33.11 & 1997 Dec. 20 & $C$ & 1800 & 1.28 & 1.6 \\
\hline & & & & & & $R$ & 900 & 1.31 & 1.6 \\
\hline \multirow[t]{2}{*}{ SL 663, LW 273, ESO 86SC22 } & 54221 & -65233 & 275.05 & -31.63 & 1999 Dec. 29 & C & 1800 & 1.43 & 1.2 \\
\hline & & & & & & $R$ & 600 & 1.48 & 1.2 \\
\hline \multirow[t]{2}{*}{ OHSC 28} & 55535 & -622043 & 271.51 & -30.24 & 1997 Dec. 23 & $C$ & 1800 & 1.33 & 1.4 \\
\hline & & & & & & $R$ & 900 & 1.36 & 1.4 \\
\hline
\end{tabular}

${ }^{a}$ Cluster identifications are from Lauberts (1982, ESO), Shapley \& Lindsay (1963, SL), Lyngå \& Westerlund (1963, LW), Kontizas et al. (1990, KMHK), Olszewski et al. (1988, OHSC).

stellar population gradually increases with galactocentric distance, from active star formation in a field at $2.3^{\circ}$ from the kinematic centre of the LMC up to an age of $1.5 \mathrm{Gyr}$ at $7.1^{\circ}$. Such a result has not been investigated within the LMC star cluster population yet. To do that, we need to enlarge the number of known clusters with ages and metallicities placed onto the same or similar scale. The fact that field stars have been formed in an outsidein process in the inner disk could be related to the aforementioned interactions between the MCs and the Galaxy and, therefore, could have a counterpart in the LMC cluster formation history. Studies of the chemical enrichment histories of field stars and clusters have arrived at a variety of results. Grocholski et al. (2006) found that the metallicity distribution of intermediate-age clusters is similar to what has been found for red giant stars in the bar, which suggests that the bar and the intermediateage clusters have similar star formation histories. Carrera et al. (2008) showed that while the disk star age-metallicity relationship (AMR) is well reproduced by closed-box models or models with a small degree of outflow, that of stars in the bar is only reproduced by models with combinations of infall and outflow. It becomes necessary to compare the AMRs of clusters and field stars using an age baseline covering the entire lifetime of the LMC.

Our group has been intensively involved in a long-term project to obtain ages and metallicities of LMC clusters, as well as investigating other important parameters. We have derived ages and metallicities for some 40 LMC clusters (Piatti et al. 2003), studied the infamous cluster age-gap (Geisler et al. 1997; Piatti et al. 2002), discovered a new giant branch clump structure (Piatti et al. 1999), searched for age and metallicity gradients (Piatti et al. 2003), and investigated in detail the AMR (Piatti et al. 2003). We continue here our previous work on LMC clusters, presenting results on five mostly unstudied clusters (NGC 1697, SL 133, NGC 1997, SL 663, OHSC 28) with the aim of adding them to our growing sample of well-studied LMC clusters that allows us to assemble a much more comprehensive database with which to study the formation and evolution of LMC clusters and their parent galaxy. As before, we employ the Washington System, initially developed for late-type stars and old stellar populations (Canterna 1976) and widely applied to intermediate age and old clusters in the Galaxy and in the Magellanic Clouds (e.g. Geisler et al. 1997; Bica et al. 1998; Geisler \& Sarajedini 1999). The long wavelength baseline covered by the $C, T_{1}$ filters can also provide a great deal of insight into the CMDs of young star clusters, especially given
Washington isochrones (Girardi et al. 2002) that allow us to determine ages. The Washington system also provides a means to determine metallicities from red giant stars.

The paper is organized as follows. The next section describes the observations and data reduction. Section 3 presents the procedure followed to estimate the cluster structural parameters, and focuses also on their CMDs along with the estimation of the cluster properties. The analysis and discussion of the results and their implications in the context of the chemical evolution of the LMC are presented in Sect. 4, and our principle findings are summarized in Sect. 5.

\section{CCD $C T_{1}$ photometry}

The observations of NGC 1997 and OHSC 28 were performed using the Tektronix $2 \mathrm{~K} \mathrm{CCD} \mathrm{\# 3} \mathrm{at} \mathrm{the} 0.9 \mathrm{~m}$ telescope at the Cerro Tololo Inter-American Observatory (CTIO) on 1997 December 20 and 23, respectively, while those of NGC 1697, SL 133 and SL 663 were obtained with the Danish Faint Object Spectrograph and Camera (DFOSC) on the $1.54 \mathrm{~m}$ Danish telescope at the European Southern Observatory (ESO) on La Silla, during the night 29 December 1999. Both the DFOSC and CTIO imagers have similar fields-of-view of $13.6 \times 13.6$ arcmin with a plate scale of $0.4^{\prime \prime} /$ pix. We used the Washington (Canterna 1976) $C$ and Kron-Cousins $R$ filters. The latter has a significantly higher throughput as compared with the Washington $T_{1}$ filter, and $R$ magnitudes can be accurately transformed to yield $T_{1}$ magnitudes (Geisler 1996).

Table 1 shows the log of the observations with filters, exposure times, airmasses, and seeing estimates. Observational setups, data reduction procedures, stellar point spread function photometry, and transformation to the standard system follow the same prescriptions described in detail in Piatti et al. (2003c, 2008). The standard star photometry shows the rootmean-square deviation of the observations from the fits to be less than $0.015 \mathrm{mag}$, indicating the nights were photometric.

The final information gathered for each cluster consists of a running star number, the CCD $x$ and $y$ coordinates, the derived $T_{1}$ magnitude and $C-T_{1}$ colour, and the photometric errors $\sigma\left(T_{1}\right)$ and $\sigma\left(C-T_{1}\right)$. Tables 2 to 6 give this information for NGC 1697, SL 133, NGC 1997, SL 663, and OHSC 28, respectively. They are only available at the CDS.

In Fig. 1, we plot - as an example - the colour-magnitude diagram (CMD) of all the measured stars in the field of NGC 1697, while Fig. 2 shows the behaviour of $\sigma\left(T_{1}\right)$ and $\sigma\left(C-T_{1}\right)$ as a 


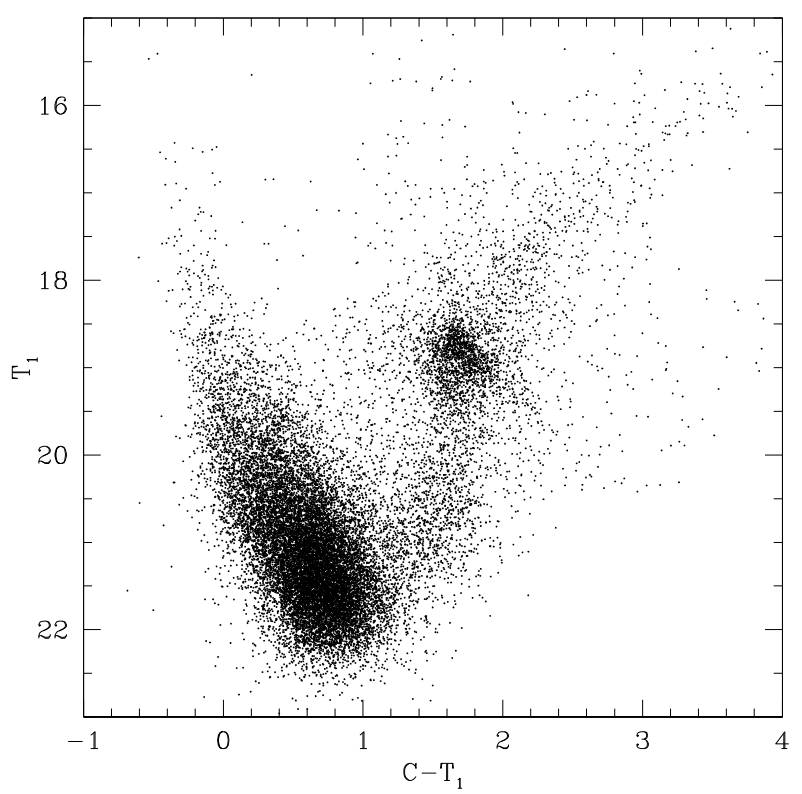

Fig. 1. $\left(C-T_{1}, T_{1}\right)$ colour-magnitude diagram for all the measured stars in the field of NGC 1697.

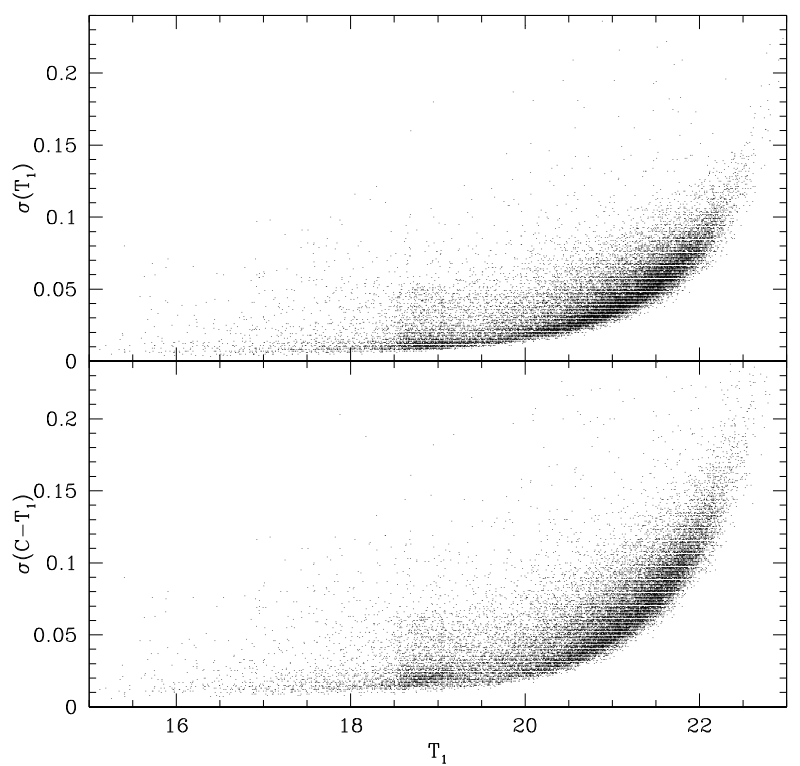

Fig. 2. $T_{1}$ magnitude and $C-T_{1}$ colour internal photometric errors as a function of $T_{1}$ for stars measured in the field of NGC 1697.

function of $T_{1}$ for this cluster. The remaining cluster fields have similar photometry quality. Note that exposures in the more critical $\mathrm{C}$ filter were longer on the smaller telescope so that the resultant photometric errors are similar. The mean magnitude and colour errors for stars brighter than $T_{1}=19$ are in the range $\left\langle\sigma\left(T_{1}\right)\right\rangle=0.015-0.030$ and $\left\langle\sigma\left(C-T_{1}\right)\right\rangle=0.015-0.040$; for stars with $T_{1}=19-21,\left\langle\sigma\left(T_{1}\right)\right\rangle \leq 0.06$ and $\left\langle\sigma\left(C-T_{1}\right)\right\rangle \leq 0.08$; and for stars with $T_{1}=21-22.5,\left\langle\sigma\left(T_{1}\right)\right\rangle \leq 0.13$ and $\left\langle\sigma\left(C-T_{1}\right)\right\rangle \leq 0.21$. The quality of our photometry allowed us to detect and measure the turn-off (TO) for all of the clusters, which was used in our age estimates. Indeed, by using the relation between the $T_{1}$ TO magnitude and age based on the theoretical isochrones by Girardi et al. (2002) and by comparing it with our data, we conclude that we are able to define TOs for stellar populations as old as $5 \pm 1 \mathrm{Gyr}\left(T_{1} \approx 22.0\right)$ with an error of $0.10 \mathrm{mag}$ in $T_{1}$.

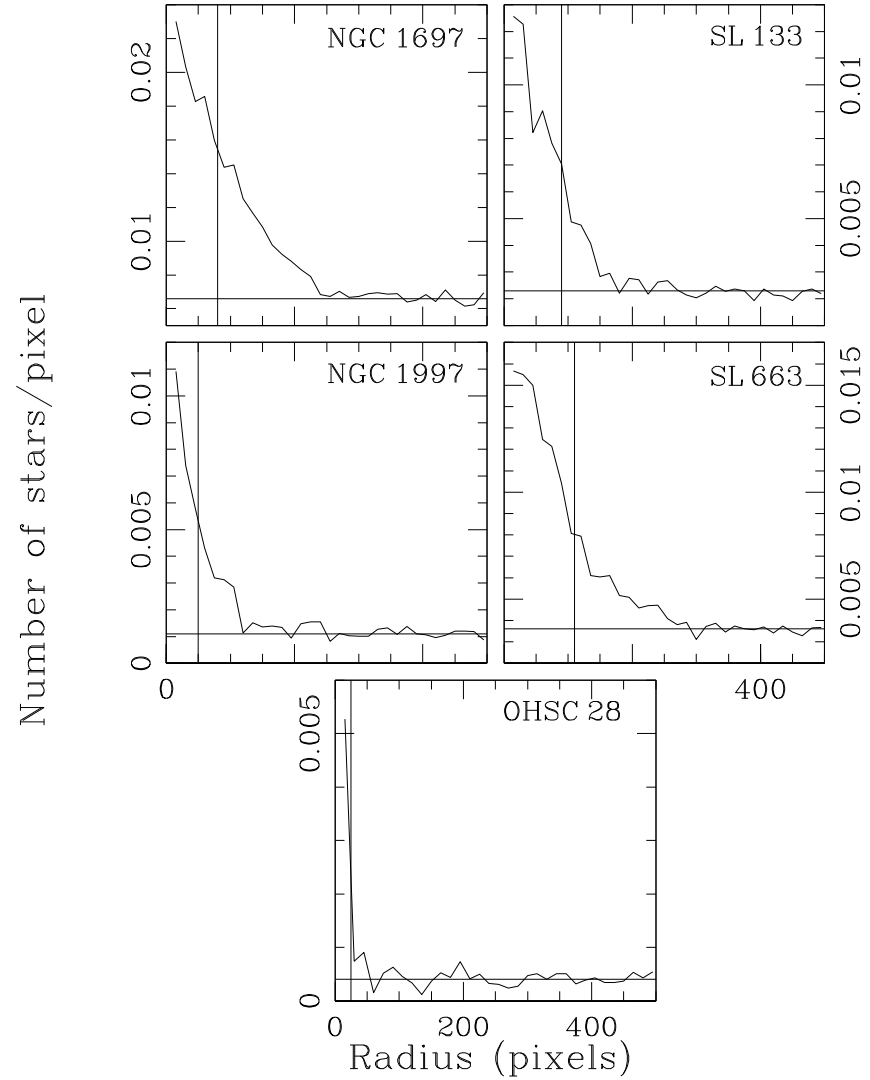

Fig. 3. Stellar density profiles for the selected clusters: NGC 1697 (upper left), SL 133 (upper right), NGC 1997 (middle left), SL 663 (middle right) and OHSC 28 (bottom). The horizontal lines correspond to the background levels far from the clusters, whereas the vertical lines indicate $r_{F W H M}$.

Slightly older and therefore fainter TOs can be reached at the expense of larger errors.

\section{Data analysis}

\subsection{Cluster properties from star counts}

In order to construct density profiles of the clusters, we began by fitting Gaussian distributions to the star counts in the $x$ and $y$ directions to determine the coordinates of the cluster centres and their estimated uncertainties. The number of stars projected along the $x$ and $y$ directions were counted using 5 pixel intervals, thus allowing us to statistically sample the spatial distributions. The fit of a single Gaussian for each projected density profile was performed using the NGAUSSFIT routine in the STSDAS/IRAF ${ }^{1}$ package. The cluster centres were determined with a typical NGAUSSFIT standard deviation of \pm 10 pixels $\left(\sim 4^{\prime \prime}\right)$.

We then constructed the cluster radial profiles by computing the number of stars per unit area at a given radius $r$, as shown in Fig. 3. The background regions of the clusters were constrained by the observed field boundaries and by a circle of radius 500 pixels from the centre of each cluster. The estimated background levels, the radii of the FWHM $\left(r_{F W H M}\right)$, and the

1 IRAF is distributed by the National Optical Astronomy Observatories, which is operated by the Association of Universities for Research in Astronomy, Inc., under contract with the National Science Foundation. 
Table 7. Cluster sizes and field contamination.

\begin{tabular}{lccccc}
\hline \hline Name & $\begin{array}{c}\text { Background }\left(\times 10^{4}\right) \\
\left(\mathrm{star} / \mathrm{px}^{2}\right)\end{array}$ & $\begin{array}{r}r_{F W H M} \\
(\mathrm{px})\end{array}$ & $\begin{array}{c}r_{\mathrm{cls}} \\
\mathrm{px})\end{array}$ & \multicolumn{2}{c}{ Field contamination $(\%)$} \\
& $66.0 \pm 7.0$ & 80 & 250 & 33 & 66 \\
\hline NGC 1697 & $23.0 \pm 5.0$ & 90 & 170 & 23 & 60 \\
SL 133 & $11.0 \pm 3.0$ & 50 & 120 & 13 & 40 \\
NGC 1997 & $36.0 \pm 6.0$ & 110 & 290 & 28 & 65 \\
SL 663 & $4.0 \pm 0.2$ & 25 & 50 & 10 & 40 \\
OHSC 28 & & & & \\
\hline
\end{tabular}

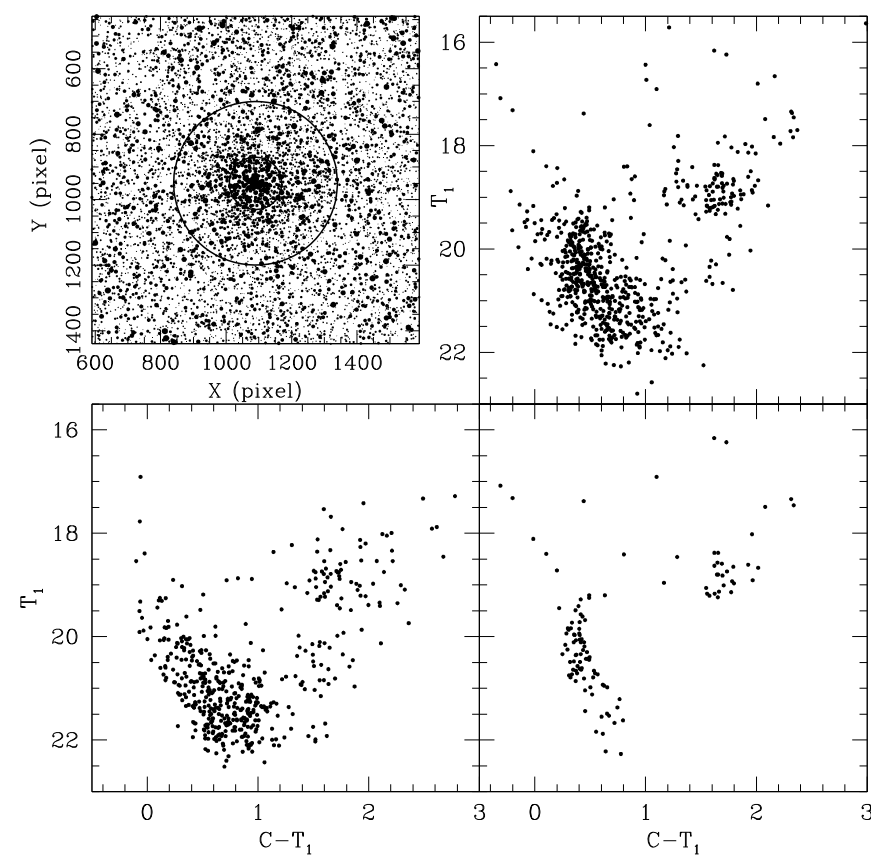

Fig. 4. Schematic finding chart of the stars observed in the field of NGC 1697 (upper left), with two concentric circles corresponding to $r_{F W H M}$ and $r_{\mathrm{cls}}$. North is up and east is to the left. The size of the plotting symbol is proportional to the $T_{1}$ brightness of the star. Three extracted CMDs for $r<r_{\mathrm{cl}}$ (upper right), the equal cluster area surrounding field (bottom left), and the cluster statistically cleaned from field contamination (bottom right).

cluster radii $\left(r_{\mathrm{cls}}\right)$ - defined as the distance from the cluster's centre where the number of stars per unit area equals that of the background - are listed in Table 7. Cluster dimensions are relatively small: 4 of the clusters have $F W H M$ between 50 and 110 pixels, while that of OHSC 28 is only 25 pixels, and they fade into the stellar field populations at a distance twice or three times $r_{F W H M}$. We then derived the statistical field star contamination for the radial intervals $r<r_{F W H M}$ and $r_{F W H M}<r<r_{\mathrm{cls}}$, yielding the values quoted in Cols. 5 and 6 of Table 7 . Note that the percentage of field stars is $1 / 10-1 / 3$ of the total number of stars in the central cluster region $\left(r<r_{F W H M}\right)$, while the contamination is $0.4-0.66$ at a radial range from $r_{F W H M}$ to $r_{\text {cls }}$.

\subsection{Cluster properties from the CMDs}

Figures 4 to 8 show three CMDs constructed using different circular extractions around each of the clusters. We also show close-up schematic finding charts of NGC 1697, SL 133, NGC 1997, SL 663, and OHSC 28 in the top left panel of the figures, respectively. In the top right panel, we show the cluster

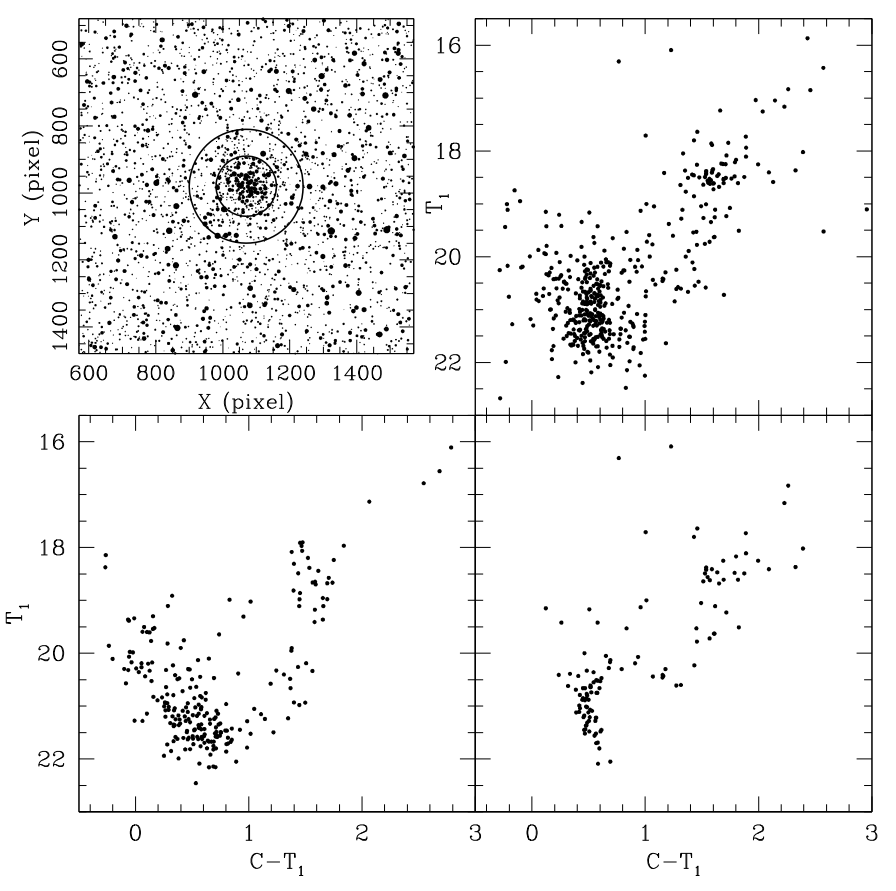

Fig. 5. Same as Fig. 4, for SL 133.

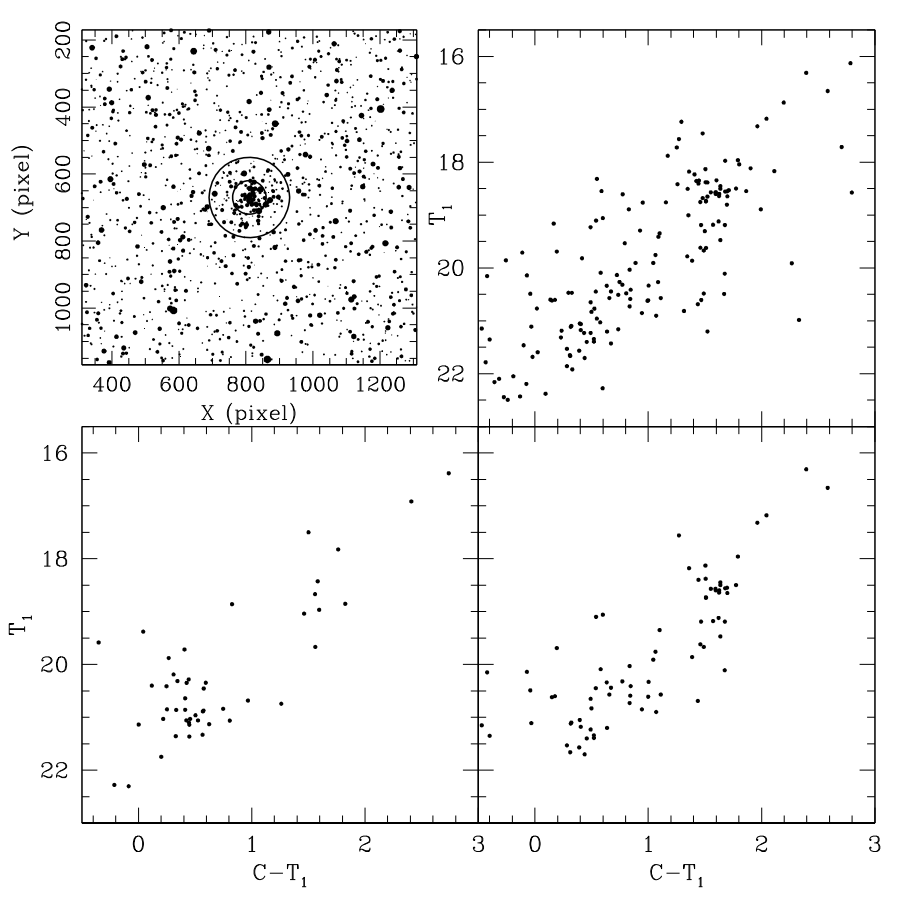

Fig. 6. Same as Fig. 4, for NGC 1997. 


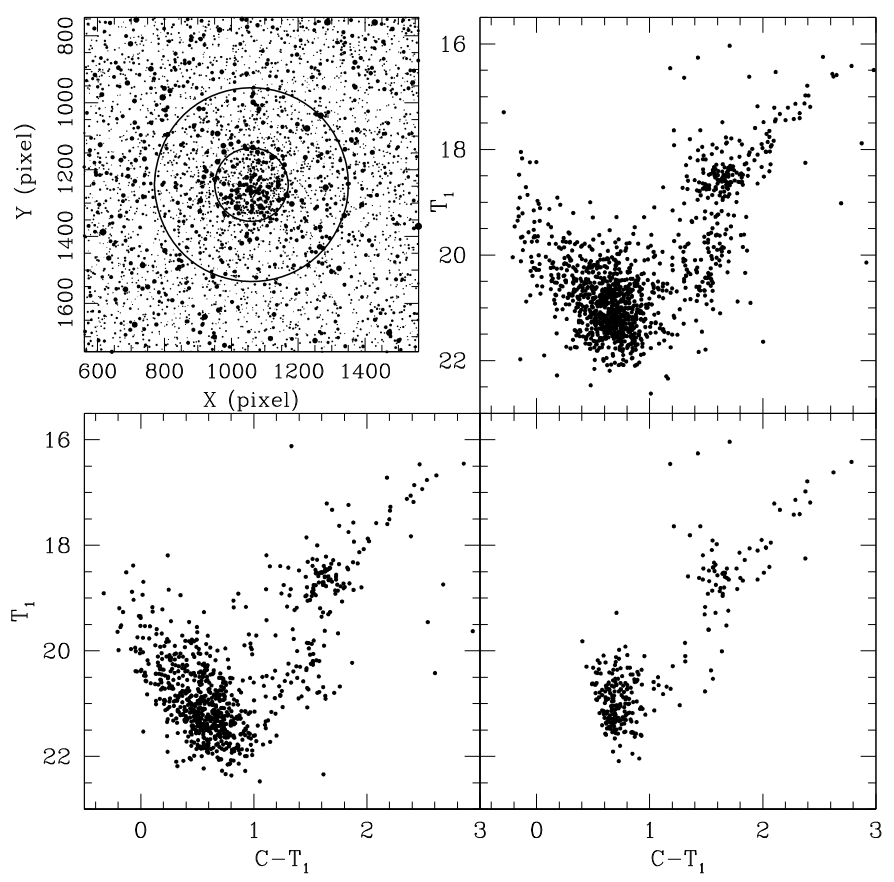

Fig. 7. Same as Fig. 4, for SL 663.

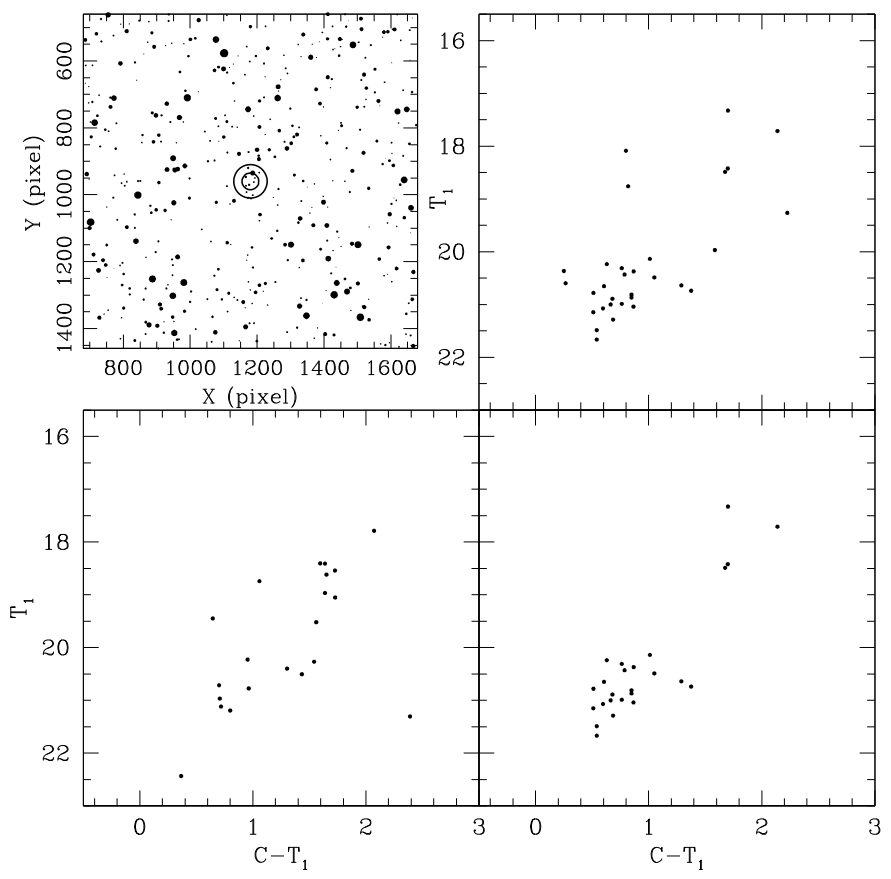

Fig. 8. Same as Fig. 4, for OHSC 28.

CMDs for stars distributed in the range $r<r_{\mathrm{cls}}$, while the right bottom panels present the corresponding cleaned cluster CMDs.

We statistically cleaned the cluster CMDs of stars that can potentially belong to the foreground/background fields or have relatively large $\sigma\left(C-T_{1}\right)$. We used four circular extractions placed well beyond the clusters, distributed throughout the observed fields, and with a total area (the sum of the four circular extraction areas) equal to that of the cluster area $\left(r=r_{\mathrm{cl}}\right)$. The resulting field CMDs, i.e. the sum of the four individual circularly extracted field CMDs, are shown in the bottom left panels of Figs. 4 to 8. We then subtracted from each cluster CMD, for different bins with sizes $\left[\Delta T_{1}, \Delta C-T_{1}\right]=(0.5,0.2) \mathrm{mag}$, the corresponding number of stars counted in the field CMDs. The total number of stars subtracted is in general agreement with the values given in Col. 2 of Table 7 . Then, we eliminated stars with $\sigma\left(C-T_{1}\right)$ larger than: 0.02 for $T_{1}<18$; 0.035 for $18 \leq T_{1}<19 ; 0.04$ for $19 \leq T_{1}<20 ; 0.055$ for $20 \leq T_{1}<20.5$; 0.065 for $20.5 \leq T_{1}<21.0$; 0.08 for $21.0 \leq T_{1}<21.5$; and 0.12 for $T_{1} \geq 21.5$, respectively. In the bottom right panels of Figs. 4 to 8 we show the CMDs of the remaining cluster stars. In the subsequent analysis, we use these CMDs for estimating the fundamental parameters of each cluster. Note that the main sequences (MSs) of the clusters appear better-defined, particularly for NGC 1697, SL 113 and Sl 663, which are immersed in relatively more crowded fields. Of course, additional sources of dispersion such as some unavoidable field interlopers, evolutionary effects and/or binarity can also take place.

Cluster reddening values were estimated by interpolating the extinction maps of Burstein \& Heiles (1982). As Table 8 lists, the $E(B-V)$ colour excesses resulted the same for four clusters. As for the cluster distance moduli, we adopt for all the clusters a value for the LMC distance modulus of $(m-M)_{0}=18.50 \pm 0.10$ (Alves et al. 2002; Pietrzynski \& Gieren 2002; Sarajedini et al. 2002), since the $T_{1}$ mag of the red giant clumps (Col. 4 of Table 8) are the same within the errors. We profited from the available theoretical isochrones computed for the Washington photometric system to estimate age of the clusters. Particularly, we used the isochrones calculated with core overshooting included by the Padova group (Girardi et al. 2002) which lead to results similar to those derived from the Geneva group's isochrones (Lejeune \& Schaerer 2001). However, the former reach fainter luminosities, allowing a better fit to the fainter portions of the MS. We used chemical compositions of $Z=$ $0.019,0.008,0.004$ and 0.001 for the isochrone sets in steps of $\Delta \log t=0.05$ dex.

We then selected a set of isochrones, along with the equations $E\left(C-T_{1}\right)=1.97 E(B-V)$ and $M_{T_{1}}=T_{1}+0.58 E(B-V)-$ $V-M_{V}$ (Geisler \& Sarajedini 1999), and superimposed them on the cluster CMDs, once they were properly shifted. In the fitting procedure, we commonly employed seven different isochrones; ranging from slightly younger to slightly older than the derived cluster age. We repeated this procedure for each one of the four metallicity values selected. Finally, from the four derived ages - corresponding to $Z=0.019,0.008,0.004$ and 0.001 , respectively - we chose the isochrone which best reproduces the cluster's main features. Columns 3 and 9 of Table 8 and Fig. 9 show the results of the isochrone fitting. For each cluster CMD, we plot the isochrone of the adopted cluster age with solid lines, and two additional isochrones bracketing the derived age with dotted lines. The ages of the bracketing isochrones were estimated by taking into account the observed dispersion (i.e., photometric errors, binarity, evolutionary effects, etc.) in the cluster CMDs. Note that the theoretically computed bluest stage during the core He-burning phase is redder than the observed red giant clump (RGC) in the CMD of NGC 1697, a behaviour which has also been detected in other studies of Galactic and Magellanic Cloud clusters (Geisler et al. 2003; Piatti et al. 2004a,b, for example).

We also derived the ages of the four clusters older than $1 \mathrm{Gyr}$ from the $\delta\left(T_{1}\right)$ index - calculated by determining the difference in the $T_{1}$ magnitude between the RGC and MS TO in the cluster CMD - and Eq. (4) of Geisler et al. (1997). The $T_{1}$ RGC and TO uncertaintes were estimated by bearing in mind the intrinsic dispersion of the cluster stars in the CMDs and the fact that the $T_{1}$ photometric errors are smaller than 0.03 and 0.06 mag for $T_{1} \sim 19$ and 21, respectively (see, Fig. 2). The mean $\delta T_{1}$ values and their errors were estimated from the average of independent 
Table 8. Fundamental parameters of LMC clusters.

\begin{tabular}{|c|c|c|c|c|c|c|c|c|c|}
\hline \multirow[t]{2}{*}{ Name } & \multirow{2}{*}{$\begin{array}{c}E(B-V) \\
(\mathrm{mag})\end{array}$} & \multicolumn{6}{|c|}{ Age } & \multicolumn{2}{|c|}{ "Metallicity } \\
\hline & & $\begin{array}{c}t_{\text {iso }} \\
(\mathrm{Gyr})\end{array}$ & $\begin{array}{c}T_{1} \text { RGC } \\
(\mathrm{mag})\end{array}$ & $\begin{array}{l}T_{1} \mathrm{TO} \\
(\mathrm{mag})\end{array}$ & $\begin{array}{l}\delta\left(T_{1}\right) \\
(\mathrm{mag})\end{array}$ & $\begin{array}{l}t_{\delta\left(T_{1}\right)} \\
(\mathrm{Gyr})\end{array}$ & $\begin{array}{c}\text { adopted } \\
\text { (Gyr) }\end{array}$ & $Z$ & {$[\mathrm{Fe} / \mathrm{H}]$} \\
\hline NGC 1697 & 0.04 & $0.70 \pm 0.10$ & - & - & - & - & $0.7 \pm 0.1$ & 0.00 & - \\
\hline SL 133 & 0.02 & $2.00 \pm 0.20$ & $18.50 \pm 0.10$ & $20.30 \pm 0.15$ & $1.80 \pm 0.25$ & $2.3 \pm 0.5$ & $2.2 \pm 0.3$ & -0.70 & $-0.65 \pm 0.20$ \\
\hline NGC 1997 & 0.04 & $2.50 \pm 0.30$ & $18.55 \pm 0.10$ & $20.50 \pm 0.20$ & $1.95 \pm 0.30$ & $2.7 \pm 0.8$ & $2.6 \pm 0.5$ & -0.70 & $-0.70 \pm 0.20$ \\
\hline SL 663 & 0.04 & $2.80 \pm 0.35$ & $18.55 \pm 0.10$ & $20.70 \pm 0.20$ & $2.15 \pm 0.30$ & $3.3 \pm 1.2$ & $3.0 \pm 0.8$ & -0.70 & $-0.75 \pm 0.20$ \\
\hline OHSC 28 & 0.04 & $2.20 \pm 0.25$ & $18.55 \pm 0.10$ & $20.50 \pm 0.20$ & $1.95 \pm 0.30$ & $2.7 \pm 0.8$ & $2.4 \pm 0.5$ & -0.70 & $-0.70 \pm 0.20$ \\
\hline
\end{tabular}

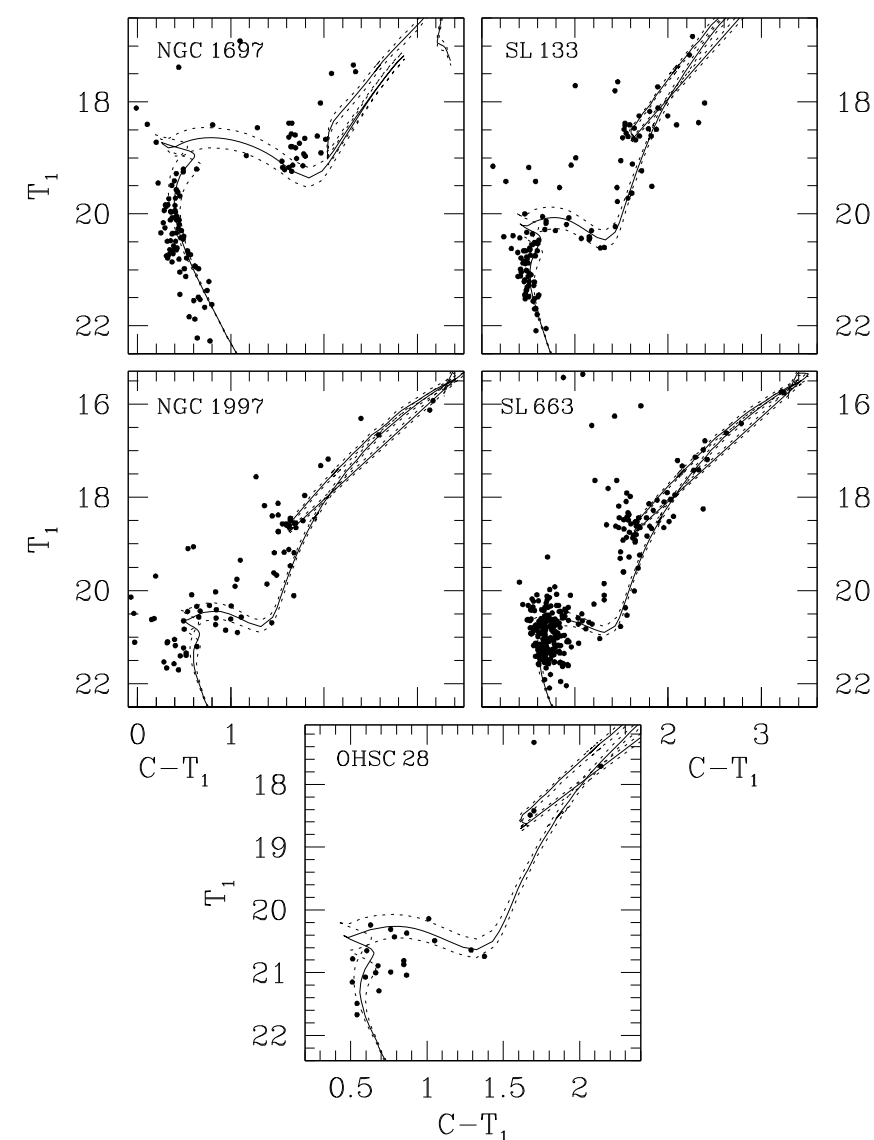

Fig. 9. Washington $T_{1}$ versus $C-T_{1}$ CMDs for the selected star clusters. Isochrones from Girardi et al. (2002), computed taking into account overshooting are overplotted. The solid and dashed lines correspond to the derived clusters ages and to the ages obtained taken into account their associated errors (see Cols. 3 and 4 of Table 8), respectively: $\log (t)=8.80,8.85$ and 8.90 and $Z=0.020$ for NGC 1697 (upper left); $\log (t)=9.25,9.30$ and 9.35 and $Z=0.004$ for SL 133 (upper right); $\log (t)=9.35,9.40$ and 9.45 and $Z=0.004$ for NGC 1997 (middle left); $\log (t)=9.40,9.45$ and 9.50 and $Z=0.004$ for SL 663 (middle right), and $\log (t)=9.30,9.35$ and 9.40 and $Z=0.004$ for OHSC 28 (bottom).

measurements by two authors. The maximum difference in $\delta T_{1}$ was only $0.2 \mathrm{mag}$, and the mean difference was $0.12 \pm 0.12 \mathrm{mag}$. Both $T_{1}$ RGC and $T_{1}$ TO, the $\delta\left(T_{1}\right)$ index and the calculated ages with their errors are succesively listed from Cols. 4 to 7 of Table 8 . We note that ages determined in this way have been found to be in good agreement with those derived from comparison to appropriate theoretical isochrones (Geisler et al. 2003; Piatti et al. 2003a,d, for example). We then averaged the ages obtained from the isochrone fitting with those derived from the
$\delta\left(T_{1}\right)$ index and adopted the final values quoted in Col. 8 of Table 8 . We assigned equal weight to both age estimates since they show good agreement, although the isochrone ages are consistently smaller. We did not derive a $\delta\left(T_{1}\right)$ age for NGC 1697 as it is too young for the calibration.

We followed the standard Standard Giant Branch (SGB) procedure of inserting absolute $M_{T_{1}}$ magnitudes and intrinsic $\left(C-T_{1}\right)_{o}$ colours for the clusters into Fig. 4 of Geisler \& Sarajedini (1999) to obtain by interpolation the cluster metal abundances $([\mathrm{Fe} / \mathrm{H}])$. These derived metallicities were corrected for age effects via the prescription given in Geisler et al. (2003). Their Fig. 6 shows that for an age of 3 Gyr, the correction results equals to 0.3 dex. The corrected metallicities for the cluster sample are listed in Col. 10 of Table 8, showing excellent agreement with the $Z$ values associated with the isochrones which best resemble the cluster features. Again, we did not perform this procedure on NGC 1697 given its young age.

Olszewsky et al. (1991) and Grocholski et al. (2006) found $[\mathrm{Fe} / \mathrm{H}]=-0.43 \pm 0.20 \mathrm{dex}$ and $-0.54 \pm 0.05 \mathrm{dex}$ for N1997 and SL 663, respectively, from the spectra of three and eight stars taken at the infrared CaII triplet. Grocholski et al. also derived metallicities for another 11 clusters which had previous estimates and found that a mean shift of $\sim 0.2$ dex exists, in the sense that their values are more metal-rich. On the other hand, bearing in mind our metallicity error and that of Grocholski et al. (see also their Fig. 12), we find good agreement between both estimates of SL 663's metal abundance.

\section{Discussion}

To investigate the chemical evolution of the LMC, we added the clusters studied herein to a list of selected LMC clusters. The selection was performed in order to generate a cluster sample with ages and metallicities determined on as similar a scale as possible to that of the present cluster sample. This avoids uncertain fundamental parameter determinations and zero-point offsets between different age and/or metallicitiy scales. All the independent age and metallicity determinations are in generally good agreement, among the 54 total LMC clusters in the sample. Note that 9 well-known old clusters were taken from the literature $(t>12 \mathrm{Gyr})$, the remaining clusters coming from our own previous studies using the same Washington $C, T_{1}$ techniques described in this paper. Table 9 lists the cluster sample along with the ages and metallicities adopted, and the deprojected angular distances assuming that all clusters are part of the inclined disk, using the standard value $i \approx 45^{\circ}$ in Westerlund (1990; see also, van der Marel \& Cioni 2001). For the galaxy centre, we adopted the position of NGC $1928\left(\alpha_{2000}=5^{\mathrm{h}} 20^{\mathrm{m}} 57^{\mathrm{s}}\right.$, $\left.\delta_{2000}=-69^{\circ} 28^{\prime} 41^{\prime \prime}\right)$. 
Table 9. Ages and metallicities of LMC clusters.

\begin{tabular}{|c|c|c|c|c|}
\hline Name & $\begin{array}{l}\text { Age } \\
\text { (Gyr) }\end{array}$ & {$[\mathrm{Fe} / \mathrm{H}]$} & $\overline{\text { Source }^{a}}$ & $\begin{array}{l}\text { Deprojected } \\
\text { distance }\left({ }^{\circ}\right)\end{array}$ \\
\hline NGC 1466 & 14.80 & -1.87 & 1,2 & 8.8 \\
\hline SL 8 & $1.80 \pm 0.30$ & $-0.40 \pm 0.20$ & 9 & 4.8 \\
\hline NGC 1697 & $0.70 \pm 0.10$ & $0.0 \pm 0.20$ & 10 & 4.0 \\
\hline NGC 1841 & 12.30 & -2.20 & 2,4 & 17.2 \\
\hline NGC 1754 & 15.50 & -1.42 & 3 & 2.6 \\
\hline SL 126 & $2.20 \pm 0.30$ & $-0.45 \pm 0.20$ & 9 & 9.8 \\
\hline SL 133 & $2.20 \pm 0.30$ & $-0.65 \pm 0.20$ & 10 & 6.5 \\
\hline NGC 1786 & 12.30 & -2.10 & 2,4 & 3.6 \\
\hline SL 218 & $0.05 \pm 0.01$ & $-0.40 \pm 0.15$ & 7 & 2.4 \\
\hline NGC 1835 & 16.20 & -1.62 & 3 & 1.7 \\
\hline NGC 1836 & $0.40 \pm 0.10$ & $0.00 \pm 0.20$ & 8 & 2.3 \\
\hline BRHT4b & $0.10 \pm 0.03$ & $-0.40 \pm 0.15$ & 7 & 2.2 \\
\hline NGC 1839 & $0.13 \pm 0.03$ & $-0.40 \pm 0.15$ & 7 & 2.2 \\
\hline NGC 1838 & $0.10 \pm 0.03$ & $-0.40 \pm 0.15$ & 7 & 2.4 \\
\hline SL 244 & $1.20 \pm 0.30$ & $-0.40 \pm 0.20$ & 6 & 2.1 \\
\hline SL 262 & $2.10 \pm 0.30$ & $-0.50 \pm 0.20$ & 9 & 9.1 \\
\hline NGC 1860 & $0.25 \pm 0.05$ & $0.00 \pm 0.20$ & 8 & 1.6 \\
\hline NGC 1863 & $0.05 \pm 0.01$ & $-0.40 \pm 0.15$ & 7 & 1.6 \\
\hline NGC 1865 & $0.50 \pm 0.10$ & $-0.20 \pm 0.20$ & 8 & 1.5 \\
\hline NGC 1898 & 13.50 & -1.37 & 3 & 0.4 \\
\hline SL 359 & $1.60 \pm 0.40$ & $-0.20 \pm 0.20$ & 6 & 1.4 \\
\hline SL 388 & $2.20 \pm 0.30$ & $-0.65 \pm 0.20$ & 9 & 7.2 \\
\hline NGC 1928 & 12.30 & -1.20 & 11 & 0.1 \\
\hline NGC 1939 & 12.30 & -2.00 & 11 & 0.7 \\
\hline SL 446a & $2.20 \pm 0.50$ & $-0.70 \pm 0.20$ & 6 & 2.0 \\
\hline SL 444 & $0.50 \pm 0.10$ & $-0.40 \pm 0.20$ & 8 & 2.1 \\
\hline SL 437 & $1.00 \pm 0.30$ & $-0.65 \pm 0.20$ & 9 & 7.3 \\
\hline SL 451 & $2.20 \pm 0.30$ & $-0.70 \pm 0.20$ & 9 & 7.5 \\
\hline SL 505 & $0.90 \pm 0.30$ & $-0.40 \pm 0.20$ & 6 & 2.9 \\
\hline SL 509 & $1.20 \pm 0.30$ & $-0.65 \pm 0.20$ & 9 & 6.6 \\
\hline LW 224 & $0.70 \pm 0.20$ & $0.00 \pm 0.20$ & 8 & 3.5 \\
\hline SL 515 & $1.60 \pm 0.30$ & - & 13 & 6.9 \\
\hline NGC 1997 & $2.60 \pm 0.50$ & $-0.70 \pm 0.20$ & 10 & 7.1 \\
\hline SL 548 & $0.40 \pm 0.10$ & $0.00 \pm 0.20$ & 8 & 3.6 \\
\hline SL 555 & $1.90 \pm 0.40$ & $-0.70 \pm 0.20$ & 6 & 3.7 \\
\hline SL 549 & $1.70 \pm 0.40$ & $-0.70 \pm 0.20$ & 6 & 5.9 \\
\hline SL 663 & $3.00 \pm 0.80$ & $-0.75 \pm 0.20$ & 10 & 4.7 \\
\hline NGC 2121 & $2.50 \pm 0.50$ & $-0.65 \pm 0.20$ & 8 & 4.3 \\
\hline OHSC 28 & $2.40 \pm 0.50$ & $-0.70 \pm 0.20$ & 10 & 8.1 \\
\hline NGC 2155 & $3.60 \pm 0.70$ & $-0.80 \pm 0.20$ & 12 & 5.4 \\
\hline SL 674 & $2.30 \pm 0.40$ & $-1.00 \pm 0.20$ & 6 & 3.9 \\
\hline SL 678 & $1.50 \pm 0.30$ & $-0.70 \pm 0.20$ & 6 & 3.9 \\
\hline SL 769 & $1.80 \pm 0.30$ & $-0.35 \pm 0.20$ & 9 & 3.9 \\
\hline SL 817 & $1.50 \pm 0.30$ & $-0.35 \pm 0.20$ & 9 & 4.7 \\
\hline ESO 121-SC03 & $8.50 \pm 0.50$ & $-1.05 \pm 0.20$ & 9 & 10.1 \\
\hline SL 842 & $2.20 \pm 0.30$ & $-0.60 \pm 0.20$ & 9 & 8.0 \\
\hline NGC 2209 & $1.50 \pm 0.30$ & $\longrightarrow$ & 13 & 8.0 \\
\hline NGC 2210 & 12.30 & -1.75 & $2,4,5$ & 5.7 \\
\hline SL 862 & $1.80 \pm 0.30$ & $-0.75 \pm 0.20$ & 9 & 6.4 \\
\hline Hodge 11 & 14.80 & -2.05 & 1,2 & 6.2 \\
\hline OHSC 33 & $1.40 \pm 0.30$ & $-0.80 \pm 0.20$ & 9 & 8.6 \\
\hline NGC 2257 & 14.80 & -1.85 & 1,2 & 8.9 \\
\hline SL 896 & $2.30 \pm 0.50$ & $-0.55 \pm 0.20$ & 12 & 7.8 \\
\hline OHSC 37 & $2.10 \pm 0.30$ & $-0.60 \pm 0.20$ & 9 & 12.4 \\
\hline
\end{tabular}

a (1) Johnson et al. (1999); (2) Carretta et al. (2000); (3) Olsen et al. (1998); (4) Brocato et al. (1996); (5) Hill et al. (2000); (6) Geisler et al. (2003); (7) Piatti et al. (2003a); (8) Piatti et al. (2003b); (9) Bica et al. (1998); (10) this work; (11) Dutra et al. (2001); (12) Piatti et al. (2002); (13) Piatti et al. (1999).

The resulting cluster list given in Table 9 contains objects distributed throughout the LMC disk and putative halo. Such a sample allows us to investigate the spatial distribution of clusters for different metallicity bins, while still keeping a

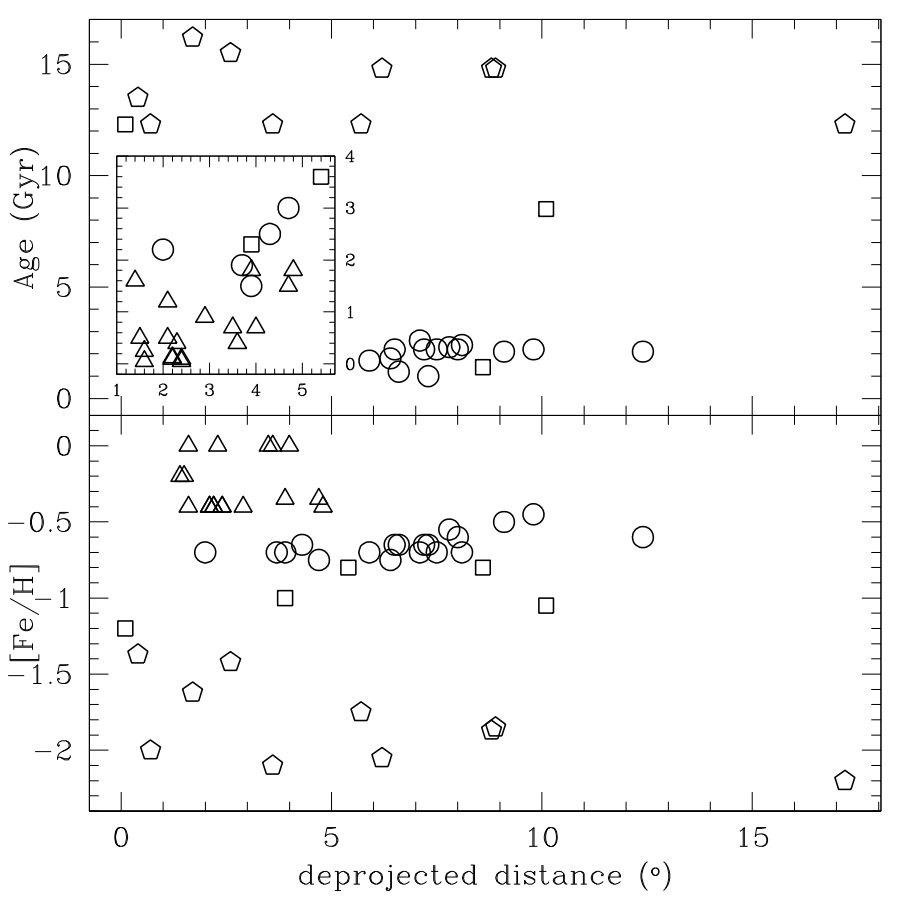

Fig. 10. Relationships between the LMC cluster ages and metallicities and the deprojected distance. Symbols correspond to different $[\mathrm{Fe} / \mathrm{H}]$ intervals: $[\mathrm{Fe} / \mathrm{H}]<-1.20$ (pentagon); $-1.20 \leq[\mathrm{Fe} / \mathrm{H}] \leq-0.80$ (square); $-0.8<[\mathrm{Fe} / \mathrm{H}]<-0.4$ (circle); $[\mathrm{Fe} / \mathrm{H}] \geq-0.4$ (triangle). The upper panel includes an enlarged view for clusters younger than $5 \mathrm{Gyr}$ old.

statistically reasonable number of objects per bin. In order to trace the cluster formation history and the metallicity enrichment of the LMC, we analyzed the cluster age-metallicity and metallicity-deprojected distance relationships. We plot in Fig. 10 the cluster ages and metallicities as a function of their deprojected angular distance for four metallicity intervals, namely: $[\mathrm{Fe} / \mathrm{H}]<-1.20$ (pentagon); $-1.20 \leq[\mathrm{Fe} / \mathrm{H}] \leq-0.80$ (square); $-0.80<[\mathrm{Fe} / \mathrm{H}]<-0.4$ (circle); and $[\mathrm{Fe} / \mathrm{H}] \geq-0.4$ (triangle), respectively. We included in the upper panel an enlargement for young clusters with deprojected distances $<6^{\circ}$.

The bottom panel of Fig. 10 reveals that the most metal-rich clusters are preferentially located in the inner disk - defined by a radius of $4^{\circ}$ (Bica et al. 1998) - where they were probably formed, since most of them are younger than 1 Gyr old (see top panel). In contrast, clusters with $[\mathrm{Fe} / \mathrm{H}]<-0.5$ exist over the entire disk out to $10^{\circ}$.

Thus, the distance from the galaxy centre alone does not appear to be the main variable to describe the cluster spatialmetallicity relationship. Our result agrees with those of Carrera et al. (2008), who concluded that the difference in mean metallicity between their innermost and the outermost fields is due to the lack of young, more metal-rich populations in the latter. Grocholski et al. (2006) also found evidence confirming that the LMC lacks the metallicity gradient typically seen in non-barred spiral galaxies, suggesting that the bar is driving the mixing of stellar populations in the LMC. However, in contrast with our Fig. 10, they found that clusters with $[\mathrm{Fe} / \mathrm{H}]>-1.0$ dex show a tight distribution $(\langle[\mathrm{Fe} / \mathrm{H}]\rangle=-0.48 \pm 0.09)$, with no tail toward solar metallicities. According to the metallicity distribution of our larger cluster sample - they observed giant stars in $28 \mathrm{LMC}$ clusters - , we find a noticeable peak at $[\mathrm{Fe} / \mathrm{H}] \sim-0.7$ dex and a notable concentration of more metal-rich clusters. However, note that the $\mathrm{Ca}$ triplet technique they employed does not work 


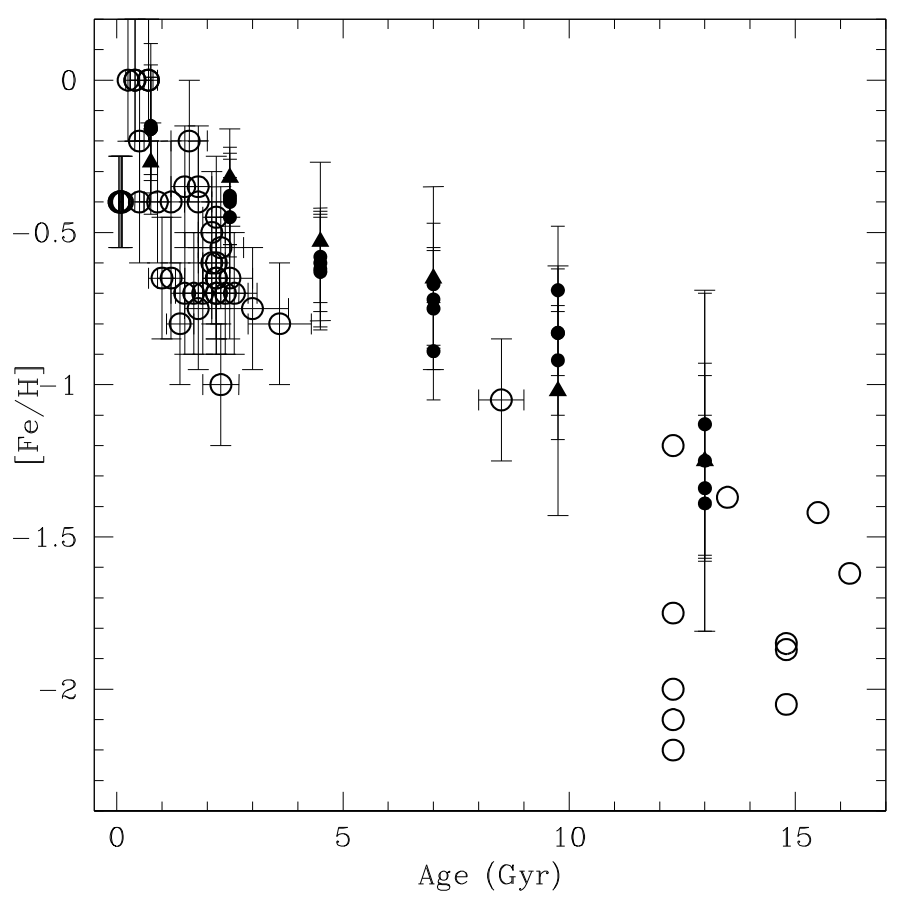

Fig. 11. Age-metallicity relation for LMC clusters (open circles) and that derived by Carrera et al. (2008, see their Table 7) for the LMC bar (filled triangles) and disk (filled circles) systems.

for clusters younger than about $1 \mathrm{Gyr}$, which are expected to be slightly more metal-rich.

The top panel of Fig. 10 shows that the most metal-poor clusters are also the oldest ones in the LMC, as expected. There also exists a quiescent period of cluster formation between 11 and 3 Gyr ago - the infamous cluster age gap (Geisler et al. 1997; Piatti et al. 2002; Bekki et al. 2004), before a large number of intermediate-age clusters (IACs) were mainly formed in the outer disk starting $\sim 3$ Gyr ago. The enlargement in the top panel shows that the subsequent cluster formation has been concentrated to the inner disk. Indeed, here a possible gradient exists, in which the younger clusters are formed closer to the galaxy centre. We find in this last result a hint for an outside-in cluster formation scenario. Gallart et al. (2008) also found that the ages of the youngest component of the dominant stellar population gradually increase with galactocentric distance, from currently active star formation in a field at $2.3^{\circ}$ from the kinematical centre of the LMC, to $1.5 \mathrm{Gyr}$ at $7.1^{\circ}$. Note that this putative outside-in formation took place in the inner disk after the bursting formation process was triggered mainly in the outer disk beginning 3 Gyr ago.

Figure 11 shows the resulting AMR, wherein open circles represent individual clusters (which carry the error bars in age and metallicity quoted in Table 9), while filled triangles and circles represent average metallicities, over the age intervals adopted by Carrera et al. (2008, their Table 7), for a sample of stars in the bar (Cole et al. 2005) and in the four different disk fields of Carrera et al. (2008), respectively. Note that the most striking difference between the disk and bar AMR found by Carrera et al. is that the disk metallicity has increased substantially $(\approx 0.22 \mathrm{dex})$ in the last $1-2 \mathrm{Gyr}$, while the bar metallicity has remained basically constant in this time interval. This different behaviour implies that, while the disk AMR is well reproduced by close-box models or models with a small degree of outflow, that of the bar is only reproduced by models with a combination of infall and outflow.

From the metal-poor end of the AMR, we find that several old clusters have $[\mathrm{Fe} / \mathrm{H}] \leq-2.2 \mathrm{dex}$, whereas the mean metallicity of the poorest field stars reach $[\mathrm{Fe} / \mathrm{H}]=-1.4$ dex. Even so, there are also old clusters within the metallicity range of the oldest field star populations. Towards the region of the IACs, we find a similar behaviour between cluster and field star abundances in the sense that the field star metallicities tend to delimit the upper envelope of the cluster metallicities. The offset between the clusters and the field population may reflect a metallicity scale difference. For ages younger than $1 \mathrm{Gyr}$ it seems that clusters and field stars share on average similar metallicity ranges. Note that in the cluster age gap, the only cluster present is at the metal-poor end of the field star distribution. In summary, we find evidence that the upper envelope of the cluster AMR appears to be coincident with that of the LMC field to within the errors, especially the disk stars. But note that Grocholski et al. (2006) showed that the metallicity distribution of intermediate-age clusters is similar to that found for red giants in the bar, which may indicate that the bar and the IACs have similar star formation histories. This result agrees, in turn, with theoretical models that suggest the bar and IACs formed as a result of a close encounter with the SMC $\sim 4$ Gyr ago (Bekki \& Chiba 2005). In this scenario, that encounter peaked at $\sim 2 \mathrm{Gyr}$ ago and triggered the formation of a large number of clusters in both MCs (Piatti et al. 2008). Recently, Bekki (2008) proposed that the MC system has a common halo, produced either by a dynamical coupling that started $\sim 4$ Gyr ago or by a remnant of a small group of galaxies destroyed via tidal stripping by the Galaxy. Unfortunately, we cannot favour either of these suggested scenarios from the available cluster data. However, since the AMR for the open clusters of the Galaxy does not show enhanced cluster formation like that which occurred in the MCs $\sim 2$ Gyr ago, perhaps both MCs have been interacting more like members of a binary system than isolated entities of a group of galaxies which includes the Milky Way.

\section{Summary and conclusions}

We have used the $0.9 \mathrm{~m}$ and $1.54 \mathrm{~m}$ telescopes at the Cerro Tololo Inter-American Observatory (CTIO) and at the European Southern Observatory (ESO), respectively, to obtain CCD imaging of a number of star clusters in the LMC as part of a continuing project. Here we have presented the CMDs of NGC 1697 , SL 133, NGC 1997, SL 663 and OHSC 28 in the Washington photometric system. The analysis of the photometric data leads to the following main conclusions:

(i) After a thorough analysis of the cluster radial density profiles, we estimated the percentage of field star contamination as a function of the distance from each cluster's centre. Field star contamination rises from $10 \%$ up to $33 \%$ at a distance corresponding to half the maximum of the cluster stellar density profile. The number of field stars reaches $40-66 \%$ at the cluster radius.

(ii) Using the observed cluster $\left(T_{1}, C-T_{1}\right)$ diagrams, statistically cleaned from field star contamination, we estimated their ages and metallicities from a comparison to theoretical isochrones and from the $\delta\left(T_{1}\right)$ index and the SGB procedure. With the exception of NGC 1697 which is a Hyadesage cluster with a solar metal content, the remaining four 
clusters are of intermediate-age (from 2.2 to $3.0 \mathrm{Gyr}$ ) and relatively metal-poor $([\mathrm{Fe} / \mathrm{H}]=-0.7 \mathrm{dex})$.

(iii) Combining these results with those for a sample of 49 additional clusters with ages and metallicities on a similar scale as the present one, we reinforce previous suggestions with respect to the chemical evolution of the LMC, that the apparent radial abundance gradient is the result of the superposition of old and intermediate-age clusters spread over the entire LMC disk, and a more metal-rich population of young clusters preferentially formed in the inner disk. These young clusters would have been formed during an outside-in process after a burst of cluster formation mainly in the outer disk that peaked $\sim 2$ Gyr ago.

(iv) Irrespective of the spatial distribution of cluster ages and metallicities and the incompleteness of our cluster sample, the cluster and field AMRs show evidence for a small offset in mean metallicity (possibly reflecting a scale difference), while overlapping on the higher metallicity envelope of the cluster AMR. Since the AMR for the open clusters of the Galaxy does not show an enhancement of cluster formation like that which occurred in the MCs $\sim 3 \mathrm{Gyr}$ ago, it is possible that the SMC and the LMC interacted more closely with each other than they did with the Galaxy.

Acknowledgements. We gratefully acknowledge the comments and suggestions of the reviewer, Dr. Fusi Pecci, which allowed us to improve the manuscript. This work was partially supported by the Argentinian institutions CONICET and Agencia Nacional de Promoción Científica y Tecnológica (ANPCyT). This work is based on observations made at the European Southern Observatory and at Cerro Tololo Inter-American Observatory (CTIO), which is operated by AURA, Inc., under cooperative agreement with the National Science Foundation. We appreciate the valuable time invested by Luis González in obtaining part of the data. A.S. acknowledges support from NSF CAREER grant AST00-94048. D.G. gratefully acknowledges support from the Chilean Centro de Astrofísica FONDAP No. 15010003 and the Chilean Centro de Excelencia en Astrofísica y Tecnologías Afines (CATA). C.G. acknowledges partial support from Chilean CONICYT through FONDECYT grant 1990638 and the Spanish Ministry of Education and Science (grant AYA2007-67913).

\section{References}

Alves, D. R., Rejkuba, M., Minniti, D., \& Cook, K. H. 2002, ApJ, 573, L51 Bekki, K. 2008, ApJ, accepted

Bekki, K., \& Chiba, M. 2005, MNRAS, 356, 680

Bekki, K., Couch, W. J., Beasley, M. A., et al. 2004, ApJ, 610, L93

Bica, E., Geisler, D., Dottori, H., et al. 1998, AJ, 116, 723
Brocato, E., Castellani, V., Ferraro, F. R., Piersimoni, A. M., \& Testa, V. 1996, MNRAS, 282, 614

Burstein, D., \& Heiles, C. 1982, AJ, 87, 1165

Canterna, R. 1976, AJ, 81, 228

Carrera, R., Gallart, C., Pancino, E., Zinn, R., \& Hardy, E. 2006, in Chemical Abundances and Mixing in Stars in the Milky Way and its Satellites, ESO Astrophysics Symposia (Springer-Verlag), 230

Carrera, R., Gallart, C., Hardy, E., Aparicio, A., \& Zinn, R. 2008, AJ, 135, 836

Carretta, E., Gratton, R. G., Clementini, G., \& Fusi Pecci, F. 2000, ApJ, 120, 1808

Cole, A. A., Tolstoy, E., Gallagher, III J. S., \& Smecker-Hane, T. 2005, AJ, 129, 1465

Dutra, C. M., Bica, E., Clariá, J. J., Piatti, A. E., \& Ahumada, A. V., 2001, A\&A, 371,895

Ferraro, I, Ferraro, F. R., Fusi Pecci, F., Corsi, C. E., \& Buonanno, R. 1995, MNRAS, 272, 391

Gallart, C., Zoccali, M., Bertelli, G., et al. 2003, AJ, 125, 742

Gallart, C., Stetson, P. B., Meschin, I. P., Pont, F., \& Hardy, E. 2008, ApJ, 682L, 89

Geisler, D. 1996, AJ, 111, 480

Geisler, D., \& Sarajedini, A. 1999, AJ, 117, 308

Geisler, D., Bica, E., Dottori, H., et al. 1997, AJ, 114, 1920

Geisler, D., Piatti, A. E., Bica, E., \& Clariá, J. J. 2003, MNRAS, 341, 771

Girardi, L., Bertelli, G., Bressan, A., et al. 2002, A\&A, 391, 195

Grocholski, A. J., Cole, A. A., Sarajedini, A., Geisler, D., \& Smith V. V. 2006, AJ, 132, 1630

Hill, V., François, P., Spite, M., Primas, F., \& Spite, F. 2000, A\&A, 364, L19

Johnson, J. A., Bolte, M., Stetson, P. B., Hesser, J. E., \& Somerville, R. 1999, ApJ, 527, 199

Kontizas, M., Morgan, D. H., Hatzidimitriou, D., \& Kontizas, E., 1990, A\&AS, 84,527

Lejeune, T., \& Schaerer, D. 2001, A\&A, 366, 538

Lyngå, G., \& Westerlund B. 1963, MNRAS, 127, 31

Olsen, K. A. G., Hodge, P. W., Mateo, M., et al. 1998, MNRAS, 300, 665

Olszewski, E. W., Harris, H. C., Schommer, R. A., \& Canterna, R. W. 1988, AJ, 95,840

Olszewski, E. W., Schommer, R. A., Suntzeff, N. B., \& Harris, H. C. 1991, AJ, 101,515

Piatti, A. E., Geisler, D., Bica, E., et al. 1999, AJ, 118, 2865

Piatti, A. E., Sarajedini, A., Geisler, D., Bica, E., \& Clariá, J. J. 2002, MNRAS, 329,556

Piatti, A. E., Geisler, D., Bica, E., \& Clariá, J. J. 2003a, MNRAS, 343, 851

Piatti, A. E., Bica, E., Geisler, D., \& Clariá, J. J. 2003b, MNRAS, 341, 977

Piatti, A. E., Clariá, J. J., \& Ahumada, A. V. 2003c, MNRAS, 340, 1249

Piatti, A. E., Bica, E., Geisler, D., \& Clariá, J. J. 2003d, MNRAS, 344, 965

Piatti, A. E., Clariá, J. J., \& Ahumada, A. V. 2004a, A\&A, 418, 979

Piatti, A. E., Clariá, J. J., \& Ahumada, A. V. 2004b, A\&A, 421, 991

Piatti, A. E., Geisler, D., Sarajedini, A., Gallart, D., \& Wischnjewsky, M. 2008, MNRAS, 389, 429

Pietrzynski, G., \& Gieren, W. 2002, AJ, 124, 2633

Shapley, H., \& Lindsay, E. M. 1963, Irish AJ, 6, 74

Sarajedini, A., Grocholski, A. J., Levine, J., \& Lada, E. 2002, AJ, 124, 2625

van der Marel, R. P., \& Cioni, M. R. L. 2001, AJ, 122, 1807

Westerlund, B. E. 1990, A\&AR, 2, 29

Whitelock, P. A., Feast, M. W., van Loon, J. Th., \& Zijlstra A. A. 2003, MNRAS, 342,86 